\title{
Eating disorders among patients with borderline personality disorder: understanding the prevalence and psychopathology
}

\author{
Mohsen Khosravi[
}

\begin{abstract}
Background: Treatment protocols can be bolstered and etiological and maintenance factors can be recognized more easily by a superior understanding of emotions and emotion regulation in the comorbidity of borderline personality disorder (BPD) and feeding and eating disorders (FEDs). Therefore, the present study aimed at investigating the prevalence and psychopathology of FEDs in patients with BPD.

Methods: In this cross-sectional study, 220 participants were examined in three groups, namely BPD $(n=38)$, BPD + FEDs $(n=72)$, and healthy controls $(n=110)$, from August 2018 to November 2019. The participants were selected by systematic random sampling among the patients who referred to Baharan psychiatric hospital in Zahedan, Iran, with the sampling interval of 3. The subjects were evaluated by 28-item General Health Questionnaire (GHQ-28), Borderline Personality Inventory (BPI), Structured Clinical Interview for DSM-5 Personality Disorders (SCID-5-PD), Structured Clinical Interviews for DSM-5: Research Version (SCID-5-RV), the 26-item Eating Attitudes Test (EAT-26), 20-item Toronto Alexithymia Scale (TAS-20), Beck Anxiety Inventory (BAI), and Beck Depression Inventory-II (BDI-II).
\end{abstract}

Results: The results showed a $65.4 \%(n=72)$ prevalence of FEDs in patients with BPD. Also, the highest and lowest prevalence rates were reported for other specified feeding and eating disorders (51.3\%) and bulimia nervosa (6.9\%), respectively. Although the highest mean score of TAS-20 was related to anorexia nervosa, there was no significant difference between the scores of various types of FEDs. The mediation analysis showed that anxiety and depression would play a mediating role in the relationship between alexithymia and eating-disordered behaviors.

Conclusions: The results have suggested that alexithymia, anxiety, and depression should receive clinical attention as potential therapeutic targets in the comorbidity of BPD and FEDs. The clinical implications of the research have been conducted to date, and directions for future research have been discussed.

Keywords: Alexithymia, Anxiety, Borderline personality disorder, Depression, Feeding and eating disorders, Psychopathology

(c) The Author(s). 2020 Open Access This article is licensed under a Creative Commons Attribution 4.0 International License, which permits use, sharing, adaptation, distribution and reproduction in any medium or format, as long as you give appropriate credit to the original author(s) and the source, provide a link to the Creative Commons licence, and indicate if changes were made. The images or other third party material in this article are included in the article's Creative Commons licence, unless indicated otherwise in a credit line to the material. If material is not included in the article's Creative Commons licence and your intended use is not permitted by statutory regulation or exceeds the permitted use, you will need to obtain permission directly from the copyright holder. To view a copy of this licence, visit http://creativecommons.org/licenses/by/4.0/. The Creative Commons Public Domain Dedication waiver (http://creativecommons.org/publicdomain/zero/1.0/) applies to the data made available in this article, unless otherwise stated in a credit line to the data. 


\section{Plain English summary}

Feeding and eating disorders (FEDs) and borderline personality disorder (BPD) are relatively common psychiatric problems amongst populations. Many people suffering from these disorders also have difficulties dealing with their emotions: they struggle to recognize and talk about their emotions (a psychological characteristic called "alexithymia") and regulate their emotions appropriately. Common comorbidity and shared psychopathology in BPD and FEDs conceptualize the relationship between eating-disordered behaviors, alexithymia, depression, and anxiety. An improved understanding of the role of emotions in the comorbidity of BPD and FEDs can help screening, enhance treatment protocols, and provide a better understanding of the etiological and maintenance factors involved in this comorbidity. Accordingly, it seems necessary to investigate the relationship between alexithymia, anxiety, depression, and eating-disordered behaviors in more detail. Therefore, the present study aimed at investigating the prevalence and psychopathology of FEDs in patients with BPD.

\section{Background}

Borderline personality disorder (BPD) is a chronic and disabling disorder, imposing many costs on societies through severe functional impairments, high risk of suicide, negative effect on the course of depressive disorders, and extensive use of treatments [1]. Clinical experience suggests that disordered-eating behavior and actual feeding and eating disorders (FEDs) are relatively common among patients with BPD such that the rate of this comorbidity has been reported at $14-53 \%$ in different studies [2-4]. Thus, this question is raised: "Is this high comorbidity an indicator of a partially causal relationship?" [5].

Despite expanded studies, the question is still unanswered [2]. In 2001, Dolan et al. [6] proposed a useful framework for developing different theoretical models to investigate the comorbidity of psychiatric disorders. For instance, the spectrum/subclinical model supposed that BPD and FEDs are similar concerning etiologies and action mechanisms $[2,6]$. Based on this assumption, it can be inferred that the use of emotion regulation strategies, including rumination, suppression, and avoidance by BPD and FEDs patients, may highlight the important role of emotion regulation (as a transdiagnostic construct) in the evolution of these disorders [7].

In this respect, researchers have indicated that alexithymia (i.e., inability to recognize and express the emotions) can be involved in many types of psychopathologies, such as BPD and FEDs, by preventing the regulation of negative emotions [8, 9]. For example, Zlotnick et al. [10] and Wolff et al. [11] indicated that patients with BPD could not usually recognize emotions and their causes.
Moreover, Nowakowski et al. [12] demonstrated that individuals with FEDs consistently reported higher levels of alexithymia on the 20-item Toronto Alexithymia Scale (TAS-20) compared to healthy controls. Accordingly, they suggested that patients used eating-disordered behaviors to control their emotional state.

Although there is evidence for the alexithymia effect on emotion regulation, little information has been obtained about this process so far [13]. Nevertheless, recent studies have suggested that unawareness of emotions results in the inability to regulate emotions successfully and, in turn, autonomic arousal is chronically increased [14]. Pandey and Mandal [15] suggested that the relationship between alexithymia and overestimated perceived arousal is due to the association of alexithymia with anxiety. Later, following the introduction of these issues, several authors considered alexithymia a personality trait that can give rise to anxiety and depression by causing problems in the management of emotions, anxiety, and depression [16]. Furthermore, the results of several studies suggested that, compared with the normal population, both anxiety and depression are significantly more prevalent among patients with comorbidity of BPD and FEDs, which can be mainly attributed to high levels of alexithymia in these patients $[12,13,16]$. In this regard, alexithymia could enhance anxiety and depression due to low self-esteem and insecurity. These secondary emotions increase the patients' distress and reduce their coping abilities, thereby leading to engagement in eating-disordered behaviors (e.g., bingeing, purging, and dietary restriction) in an effort to avoid or cope with the emotions $[12,16,17]$.

Thus, given that FEDs is a subtype of emotional disorders, deficits in emotional processing observed in FEDs should be fully mediated by anxiety and depression. In other words, if FEDs do not constitute a distinct pathology from emotional disturbances, only anxiety and depression must mediate the relationship between alexithymia and eating-disordered behaviors [18, 19]. Consistent with the complete mediation hypothesis, Eizaguirre et al. [16] revealed that anxiety and depression can mediate the relationship between alexithymia and eating-disordered behaviors.

Moreover, an improved understanding of the role of emotions in the comorbidity of BPD and FEDs can help screening, enhance treatment protocols, and provide a better understanding of the involved etiological and maintenance factors. Therefore, it seems necessary to investigate the relationship between alexithymia, anxiety, depression, and eating-disordered behaviors in further detail. For this purpose, the present research pursues three goals, including (1) comparing the mean scores of Beck Anxiety Inventory (BAI), Beck Depression Inventory-II (BDI-II), TAS-20, the 26-item Eating 
Attitudes Test (EAT-26), and 28-item General Health Questionnaire (GHQ-28) for the three study groups (including BPD, BPD + FEDs, and healthy controls); (2) comparing the mean scores of TAS-20 in terms of different FEDs subtypes in BPD + FEDs patients; (3) investigating the mediating role of anxiety and depression in the relationship between alexithymia and eatingdisordered behaviors in both BPD only and BPD + FEDs groups.

\section{Methods}

\section{Participants}

This cross-sectional study was performed from August 2018 to November 2019. In this study, 110 patients with BPD and 110 healthy people were selected. The sample size was calculated based on the study performed by Eizaguirre et al. [16]. Power calculations for $80 \%$ power to detect and effect size of $r=0.3$ at alpha $=0.5$ yielded a necessary sample of $N=220$ [20]. The patients with BPD were selected by systematic random sampling among the persons who referred to Baharan psychiatric hospital in Zahedan, Iran, with the sampling interval of 3. Also, the healthy people were selected out of their relatives through one-to-n matching (i.e., one case dedicated to one control). The inclusion criteria were as follows: (1) getting a score above 10 in Borderline Personality Inventory (BPI) and approved diagnosis of the disorder based on Structured Clinical Interview for DSM-5 Personality Disorders (SCID-5-PD) by a psychiatrist; (2) age range of 18-35 years; (3) ability to read and write alongside reading comprehension; (4) for healthy people, getting a score of $<21$ in GHQ-28 and approved mental health based on Structured Clinical Interviews for DSM-5: Research Version (SCID-5-RV) and SCID-5-PD by the psychiatrist. The exclusion criteria included the followings: (1) severe and acute physical illness; (2) brain traumatic injury; (3) comorbidity of schizophrenia and other psychotic disorders; (4) epileptic disorder; (5) intellectual disability; (6) mixed personality disorder; (7) taking any drug or substance that causes anorexia and bulimia; (8) failing to fill the questionnaires properly.

In this study, 220 participants were evaluated in three groups, namely BPD $(n=38)$, BPD + FEDs $(n=72)$, and healthy controls $(n=110)$. Table 1 presents the participants' sociodemographic information. According to the table, there was no significant difference in sociodemographic factors between the study groups.

\section{Procedures}

After the approval of the research project by the ethics committee of the Medical Faculty of the Zahedan University of Medical Sciences (IR.ZAUMS.REC.1398.212), the informed consent forms were distributed among the participants. In order to follow the Declaration of
Helsinki, participation in the study was optional, and the participants could leave the study for any reason. After obtaining informed consent from the participants, GHQ-28, EAT-26, TAS-20, BAI, and BDI-II were distributed among them. Next, the psychiatrist evaluated all of the participants using SCID-5-PD and SCID-5-RV to identify the three study groups (including BPD, BPD + FEDs, and healthy controls). The questionnaires were anonymous to keep the participants' information private.

\section{Measures}

The following measures were used in this study (in general, the Cronbach's alpha values of 0.70 or higher indicate acceptable internal consistency) [21]:

\section{TAS-20}

Alexithymia was assessed with the Persian version of the TAS-20, a 20-item self-report questionnaire scored based on a five-point (1-5) Likert scale. The minimum and maximum scores are 20 and 100, respectively, and scores of $\geq 61$ represent alexithymia [22]. In this study, the Cronbach's alpha coefficients for the TAS-20 subscales of difficulty identifying feelings, difficulty describing feelings, and externally oriented thinking were 0.85 , 0.82 , and 0.75 , respectively, while it was 0.72 for the total scale.

\section{$B A I$}

Anxiety symptoms were assessed with the Persian version of the BAI, a self-report 21-item questionnaire scored based on a four-point (0-3) Likert scale. The minimum and maximum scores are 0 and 63 , respectively [23]. In this study, the Cronbach's alpha coefficient for the BAI was 0.90 .

\section{BDI-II}

Depressive symptoms were assessed with the Persian version of the BDI-II, a self-report 21-item questionnaire scored based on a four-point (0-3) Likert scale. The minimum and maximum scores are 0 and 63, respectively [24]. In this study, the Cronbach's alpha coefficient for the BDI-II was 0.88 .

\section{EAT-26}

Eating-disordered behaviors were assessed with the Persian version of the EAT-26. In this 26-item questionnaire, the minimum and maximum scores are equal to 0 and 78 , respectively. A score above 20 stands for the probability of being affected by FEDs [25]. In this study, the Cronbach's alpha coefficients for the EAT-26 subscales were as follows: drive for thinness $=0.90$, restrained eating $=0.77$, perceived social pressure to eat $=$ 0.87 , food preoccupation and oral control $=0.75$ and bulimia $=0.71$. 
Table 1 Comparisons of the socio-demographic factors, BAI, BDI-II, TAS-20, EAT-26, and GHQ-28 among the three study groups $(N=220)$

\begin{tabular}{|c|c|c|c|c|c|c|}
\hline Variables & Subvariables & $\begin{array}{l}\text { Healthy Controls }(n= \\
\text { 110) }\end{array}$ & $\begin{array}{l}\mathrm{BPD}(n= \\
38)\end{array}$ & $\begin{array}{l}\text { BPD }+ \text { FEDs }(n= \\
72)\end{array}$ & & \\
\hline & & $n(\%)$ & $n(\%)$ & $n(\%)$ & $x^{2}(2)$ & \\
\hline & & 37 (33.6) & $10(26.3)$ & $23(31.9)$ & 0.71 & \\
\hline \multirow[t]{3}{*}{ Age } & $18-23$ & & & & & \\
\hline & $24-29$ & $43(39.1)$ & $16(42.1)$ & $26(36.1)$ & & \\
\hline & $30-35$ & $30(27.3)$ & $12(31.6)$ & $23(31.9)$ & & \\
\hline \multirow[t]{2}{*}{ Gender } & Male & $28(25.5)$ & $17(44.7)$ & $26(36.1)$ & 5.26 & \\
\hline & Female & $82(74.5)$ & $21(55.3)$ & $46(63.9)$ & & \\
\hline \multirow[t]{6}{*}{ Marital status } & Never married & $57(51.8)$ & $22(57.9)$ & $34(47.2)$ & 17.71 & \\
\hline & Married & $28(25.5)$ & $16(42.1)$ & $18(25.0)$ & & \\
\hline & Cohabiting & $5(4.5)$ & $0(0)$ & $2(2.8)$ & & \\
\hline & Widowed & $2(1.8)$ & $0(0)$ & $5(6.9)$ & & \\
\hline & Divorced & $13(11.8)$ & $0(0)$ & $8(11.1)$ & & \\
\hline & Separated & $5(4.5)$ & $0(0)$ & $5(6.9)$ & & \\
\hline \multirow{3}{*}{$\begin{array}{l}\text { Education } \\
\text { level }\end{array}$} & Non-degree & $45(40.9)$ & $9(23.7)$ & $28(38.9)$ & 1.84 & \\
\hline & $\begin{array}{l}\text { High school } \\
\text { diploma }\end{array}$ & $38(34.5)$ & $4(10.5)$ & $23(31.9)$ & & \\
\hline & Academic degree & $27(24.5)$ & $25(65.8)$ & $21(29.2)$ & & \\
\hline \multirow[t]{3}{*}{ Income } & $<10,000,000$ Rials & $73(66.4)$ & $23(60.5)$ & $44(61.1)$ & 0.70 & \\
\hline & $\geq 10,000,000$ Rials & 37 (33.6) & $15(39.5)$ & $28(38.9)$ & & \\
\hline & & $M(S D)$ & M (SD) & $\mathrm{M}(\mathrm{SD})$ & $F(2,217)$ & Scheffé post-hoc test \\
\hline $\mathrm{BAl}$ & & $7.94(6.20)$ & $50.65(8.91)$ & $62.30(2.86)$ & $2005.08^{* * *}$ & $\begin{array}{l}\mathrm{BPD}+\mathrm{FEDs}>\mathrm{BPD}>\text { Healthy } \\
\text { Controls }\end{array}$ \\
\hline BDI-II & & $3.53(5.62)$ & $26.07(4.46)$ & $38.90(6.40)$ & $866.85^{* * *}$ & $\begin{array}{l}\mathrm{BPD}+\mathrm{FEDs}>\mathrm{BPD}>\text { Healthy } \\
\text { Controls }\end{array}$ \\
\hline TAS-20 & & $28.10(7.18)$ & $\begin{array}{l}53.94 \\
(12.95)\end{array}$ & $79.00(9.60)$ & $671.54^{* * *}$ & $\begin{array}{l}\mathrm{BPD}+\mathrm{FEDS}>\mathrm{BPD}>\text { Healthy } \\
\text { Controls }\end{array}$ \\
\hline EAT-26 & & $1.32(2.11)$ & $8.55(5.19)$ & $28.08(8.45)$ & $521.03^{* * *}$ & $\begin{array}{l}\text { BPD }+ \text { FEDs }>\text { BPD }>\text { Healthy } \\
\text { Controls }\end{array}$ \\
\hline GHQ-28 & & $10.10(5.48)$ & $36.73(7.09)$ & $58.70(11.30)$ & $797.78^{* * *}$ & $\begin{array}{l}\text { BPD }+ \text { FEDs }>\text { BPD }>\text { Healthy } \\
\text { Controls }\end{array}$ \\
\hline
\end{tabular}

Note. BAI Beck Anxiety Inventory, BDI-II Beck Depression Inventory-II, BPD Borderline personality disorder, EAT-26 26-item Eating Attitudes Test, FEDs Feeding and eating disorders, TAS-20 20-item Toronto Alexithymia Scale. ${ }^{*} p<0.05 ;{ }^{* *} p<0.01 ;{ }^{* * *} p<0.001$.

BPI

In this 53-item questionnaire (answered by yes or no), if the person's score for the 20 items of the cutoff score is above 10 , the person is most likely to be affected by BPD [26]. In this study, the Cronbach's alpha coefficients for the BPI subscales of identity diffusion, primitive defense mechanisms, reality testing, and fear of closeness were $0.78,0.82,0.74$, and 0.77 , respectively, while it was 0.80 for the total scale.

\section{$S C I D-5-P D$}

SCID-5-PD is a semi-structured clinical interview used by researchers and clinicians, which evaluates DSM-5 personality disorders under three clusters of $\mathrm{A}, \mathrm{B}$, and $\mathrm{C}$, and other specific personality disorders. Several studies have reported acceptable reliability and validity of SCID5-PD [27].

\section{$S C I D-5-R V$}

SCID-5-RV is a semi-structured interview for major DSM-5 diagnoses, which is performed by a trained clinician or health expert familiar with the diagnostic criteria and classification of disorders in DSM-5. Several studies have reported acceptable reliability and validity of SCID5-RV [28].

\section{GHQ-28}

GHQ-28 is a 28-item questionnaire in which items are scored in the range of $0-3$. The overall score ranges 
between 0 and 84 . A score of $<21$ indicates a person's mental health [29]. In this study, the Cronbach's alpha coefficients for the GHQ-28 subscales of somatic symptoms, anxiety and insomnia, social dysfunction, and severe depression were $0.76,0.84,0.71$, and 0.88 , respectively, while it was 0.90 for the total scale.

\section{Data analysis}

Statistical analysis was performed using descriptive statistics, including mean and standard deviation. Chi square test and Kruskal-Wallis test were conducted for a sociodemographic comparison of the three study groups. Also, an analysis of variance (ANOVA) was used to compare the mean scores of BAI, BDI-II, TAS-20, EAT26, and GHQ-28. In ANOVA, the Scheffé test was applied to the post-hoc analysis. Subsequently, in BPD + FEDs group, the Pearson correlation coefficient was used to evaluate the correlation between the study variables. Also, in this group, the mediation analysis was conducted to investigate the mediating effect of anxiety and depression on the relationship between alexithymia and eating-disordered behaviors using the Hayes' PROCESS macro for SPSS [30]. As outlined in Preacher and Hayes [31], mediation emerges when the indirect effect is significant and the confidence intervals do not contain zero. Furthermore, given the relationship of sociodemographic factors (including age, gender, marital status, education level, and income) with anxiety, depression [32-35], and FEDs [36, 37] obtained in previous studies, the above-mentioned factors were considered as covariates in the mediation analysis. Meanwhile, data analysis was performed by SPSS 25, and the significance level was set at $P<0.05$.

\section{Results}

Preliminary analysis

According to Table 1, there was a significant difference between the scores of BAI, BDI-II, TAS-20, EAT-26, and GHQ-28 among the three study groups. Based on Scheffé post-hoc test, the highest scores of BAI, BDI-II, TAS-20, EAT-26, and GHQ-28 were reported for BPD + EFD group, and the lowest scores were reported for healthy controls.

The results of the SCID-5-RV interview indicated a 65.4\% current prevalence of FEDs in patients with BPD. The highest and lowest frequencies were reported for other specified feeding and eating disorders $(51.3 \%)$ and bulimia nervosa (6.9\%), respectively (Fig. 1). No FEDs case was observed in the BPD only group through clinical interviewing. Although the mean scores of TAS-20 in the BPD + FEDs group were significantly higher than those of the BPD only group, this difference was not significant in terms of different types of FEDs (Fig. 2).

\section{Associations of study variables}

The results of correlation in both the BPD only and BPD + FEDs groups suggested a significant positive correlation between the scores of EAT-26 and BAI $(\mathrm{r}=0.58$; $p<0.01)$, BDI-II $(\mathrm{r}=0.52 ; p<0.01)$, and TAS-20 $(\mathrm{r}=$ 0.59; $p<0.01$ ). Furthermore, there was a significant positive correlation between the scores of TAS-20 and BAI $(\mathrm{r}=0.67 ; p<0.01)$ and BDI-II $(\mathrm{r}=0.52 ; p<0.01)$ (see Table 2).

\section{Mediation analysis}

The mediation analysis was performed using Hayes' PROCESS tool in SPSS (Model $=4$, Bootstrap

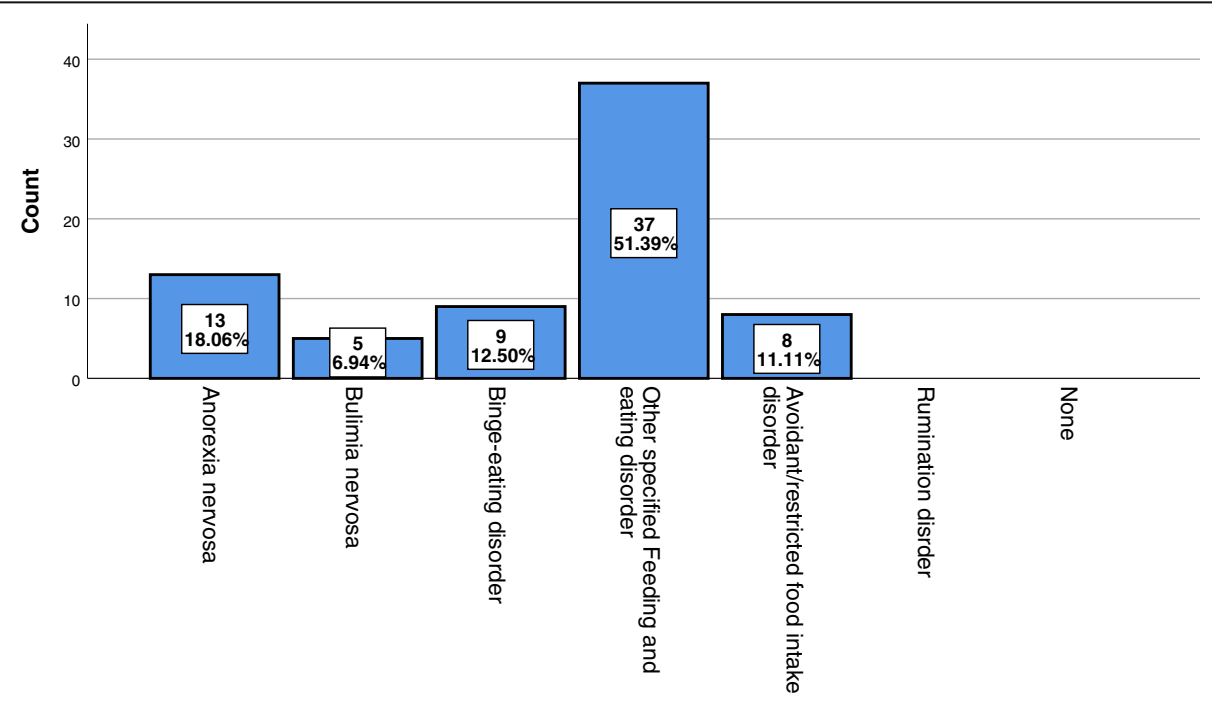

Fig. 1 The prevalence of different types of FEDs in BPD + FEDs group $(n=72)$. Note. BPD: borderline personality disorder; FEDs: Feeding and eating disorders 


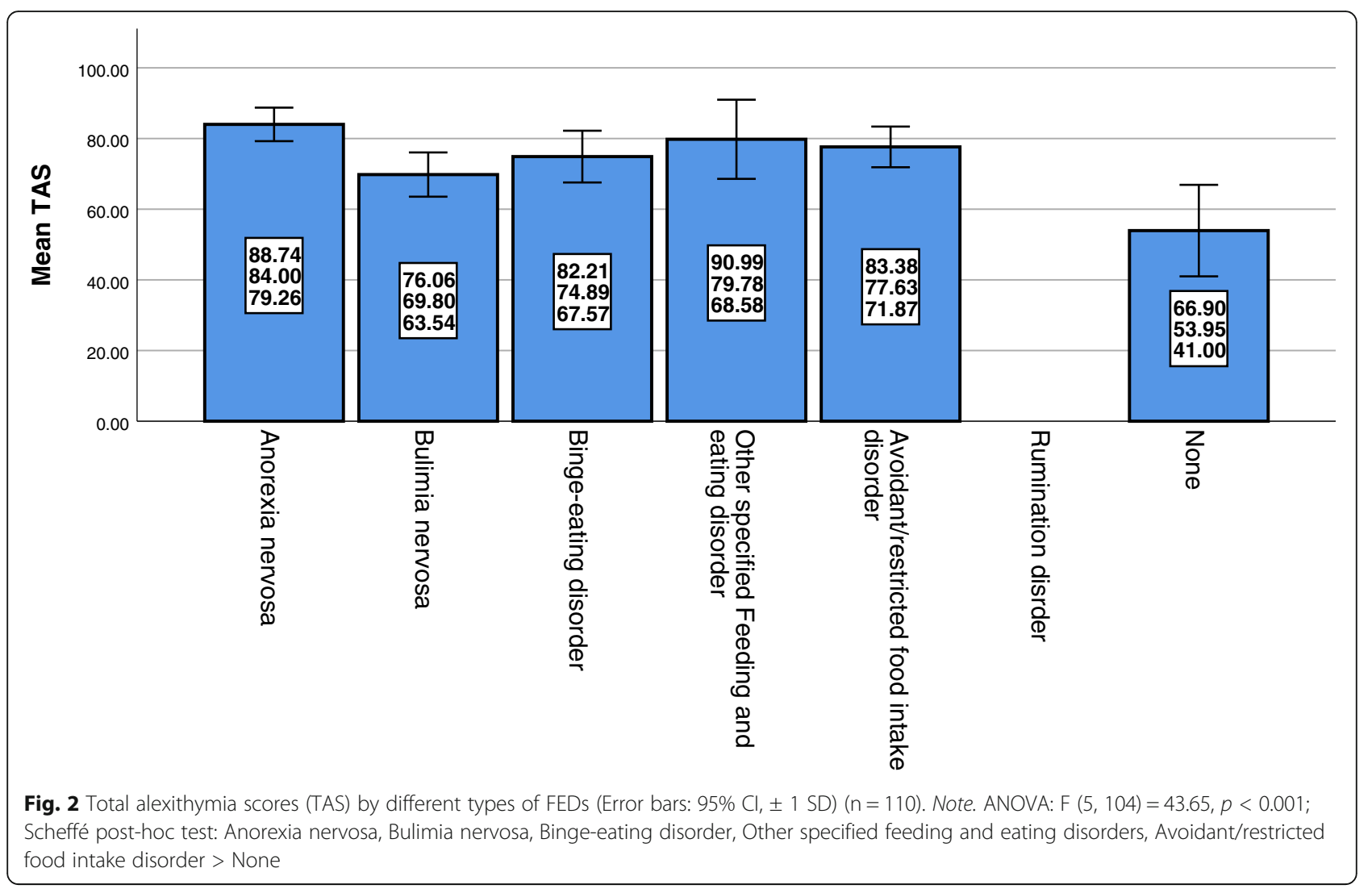

Samples $=5000$ ). As hypothesized, a significant indirect effect of Alexithymia was direst observed on eating-disordered behaviors through anxiety and depression $(\beta=0.12,95 \%$ CI: $0.005,0.26 ; \beta=0.10,95 \%$ CI: $0.004,0.18$, respectively). The mediators (i.e., anxiety and depression) could account for roughly $54 \%$ $\left(\mathrm{P}_{\mathrm{M}}=0.54\right)$ and $45 \%\left(\mathrm{P}_{\mathrm{M}}=0.45\right)$ of the total effect of alexithymia on EAT-26 total score, respectively. Hence, the overall hypothesis that anxiety and depression mediate the effect of alexithymia on eatingdisordered behaviors was supported (Fig. 3).

\section{Discussion}

In this study, there was a significant difference in the scores of BAI, BDI-II, TAS-20, EAT-26, and GHQ-28

Table 2 Correlations between BAI, BDI-II, TAS-20, and EAT-26 in BPD + FEDs group $(n=72)$

\begin{tabular}{llllll}
\hline Variables & M (SD) & 1 & 2 & 3 & 4 \\
\hline 1. BAI & $58.69(7.59)$ & 1 & & \\
2. BDI-II & $34.76(8.35)$ & $0.53^{* *}$ & 1 & \\
3. TAS-20 & $71.06(15.31)$ & $0.67^{* *}$ & $0.52^{* *}$ & 1 \\
4. EAT-26 & $22.71(12.03)$ & $0.58^{* *}$ & $0.52^{* *}$ & $0.59^{* *}$ & 1 \\
\hline
\end{tabular}

Note. BAI Beck Anxiety Inventory, BDI-II Beck Depression Inventory-II, EAT-26 26-item Eating Attitudes Test, TAS-20 20-item Toronto Alexithymia Scale ${ }^{*} p<0.05 ;{ }^{* *} p<0.01 ;{ }^{* * *} p<0.001$ among the three study groups. The highest scores were reported for the BPD + FEDs group, and the lowest scores were obtained for healthy controls. These findings were consistent with the results of the study performed by Eizaguirre et al. [16], Nowakowski et al. [12], and Gilboa-Schechtman et al. [18] who showed that the scores of TAS-20, BAI, and BDI-II in the BPD + FEDs group are higher than those of healthy controls. These results indicated that the BPD + FEDs patients had more difficulties in identifying and describing their emotions than BPD only patients and healthy controls potentially due to a greater degree of distress and less effective coping styles $[12,16]$.

Additionally, in this study, a higher FEDs prevalence was reported among patients with BPD compared to previous studies $(65.4 \%$ vs. $14-53 \%)$. The highest and the lowest frequencies were reported for other specified feeding and eating disorders (51.3\%) and bulimia nervosa $(6.9 \%)$, respectively. In line with the findings, in the study performed by Zanarini et al. [2, 38], eating disorders not otherwise specified (i.e., diagnosis of other specified feeding and eating disorders in DSM-5) were the most common FEDs among patients with BPD. Moreover, the low rate of bulimia nervosa in patients with BPD has been contrary to much prior research [2-4, 38]. This is because FEDs frequency among patients with BPD has been investigated based on DSM-III-R and 


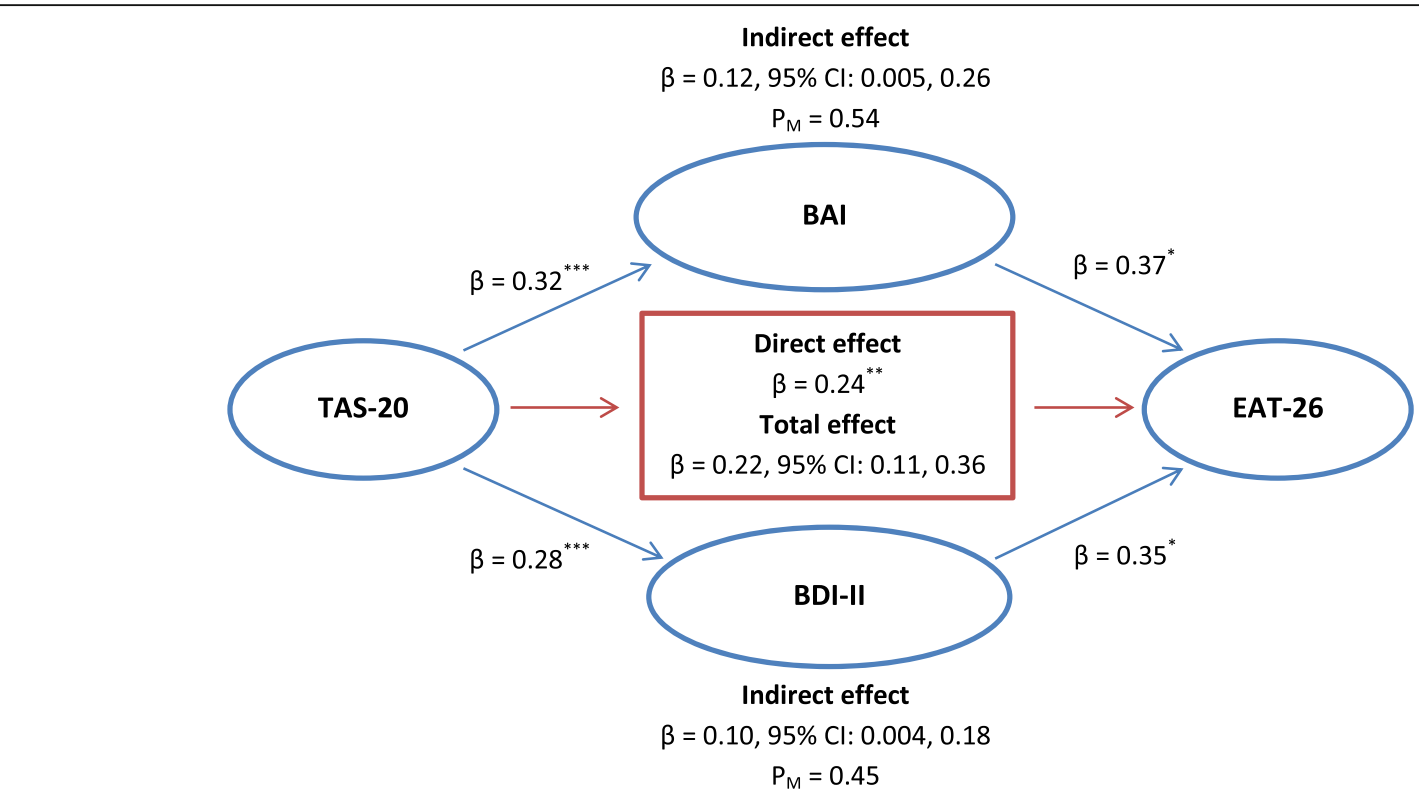

Fig. 3 Illustration of the results of the mediation analysis described in the text, which tested BAI and BDI-II total scores (the measures of anxiety and depression, respectively) as the potential mediators of the relationship between TAS-20 and EAT-26 total scores (the measures of alexithymia and disordered eating behaviors, respectively) by controlling for sociodemographic variables (included age, gender, marital status, degree level, and income) in BPD + FEDs group $(n=72)$. Note. PM: Effect size (ratio of indirect to total effect) ${ }^{*} p<0.05 ;{ }^{* * *} p<0.01 ;{ }^{* * *} p<0.001$.

DSM-IV-TR criteria in previous studies, which are different from DSM-5 criteria. In addition, avoidant/restricted food intake disorder is a new diagnosis in DSM5 that is very similar to anorexia nervosa, aside from the fact that there is no distress about body shape/size or fear of fatness in avoidant/restricted food intake disorder [28]. No subcategory of FEDs was observed in the only BPD group trough interviewing, suggesting that EAT-26 can be assumed as a useful screening instrument (with a cutoff point of above 20) [25] for diagnosing different types of FEDs in patients with BPD.

Although the highest mean score of TAS-20 in this study was related to anorexia nervosa, there was no significant difference between the scores of different types of FEDs. This finding was consistent with the results of the studies performed by Cochrane et al. [9], Troop et al. [39], and Berthoz et al. [40]. However, it was inconsistent with the studies performed by Eizaguirre et al. [16], Nowakowski et al. [12], and Gilboa-Schechtman et al. [18], who showed that mean score of TAS-20 in patients with anorexia nervosa was significantly higher than that in other types of FEDs. However, the findings may reflect insufficient power as there were not many anorexia nervosa cases in the study sample. Thus, it may represent a Type 2 error rather than a sign that anorexia nervosa does not present with high alexithymia.

Together the results demonstrated a considerable positive correlation between the scores of EAT-26 and BAI, BDI-II, and TAS-20. Moreover, there was a significant positive correlation between the scores of TAS-20 and BAI and BDI-II. These findings suggested that, in patients affected by BPD + FEDs, alexithymia was closely related to anxiety and depression. Here, this question is raised: "Is alexithymia either a state variable or a trait variable?" There is strong evidence that alexithymia is not only a by-product of FEDs symptomatology. However, there is mixed evidence of whether alexithymia is independent of general distress $[12,16,18]$. Nevertheless, several studies have indicated that elevated alexithymia scores become eliminated by controlling the effect of distress. However, some other studies have suggested that, in patients with FEDs, elevated alexithymia scores remain constant even by controlling the effect of anxiety and depression [12]. In this study, the mediation analysis showed that the role of alexithymia in the prediction of eating-disordered behaviors remained unchanged even by controlling the effect of anxiety and depression. Accordingly, alexithymia may be a trait rather than a state. In this regard, Schmidt et al. [41] stated that pharmacotherapy with antidepressants can only decrease depression level in patients with high levels of alexithymia and depression. Moreover, genetic studies have concluded that alexithymia has its own heritability component that cannot be fully explained by depression or genetic susceptibility to general distress and psychopathology [42]. As a result, although the relevant evidence is mixed, it is recommended that clinical attention should be paid to alexithymia as a distress-independent construct [12]. 
However, the cross-sectional design of this study prevents an understanding of relationships' exact nature, particularly with respect to causality.

The findings have many implications for nosology and interventions. First, the FEDs among patients with BPD are likely to be a subtype of emotional disorders that, in turn, should affect the prediction of the course of these afflictions. For example, if depressive episodes as a primary disorder constitute the core of these conditions, the risk of developing depressive episodes remains high, even with the recovery from the FEDs. Second, this nosological shift may also affect the type of treatment. If anxiety and depression, for instance, are diagnosed as primary or secondary disorders, the treatment will be different. Third, emotional awareness normalization and emotion regulation may be regarded as recovery markers beyond symptom reduction [18].

However, there were some limitations faced in this study. First, the cross-sectional design avoided the precise understanding of relationships' nature, especially concerning causality. It is essential to differentiate between primary and secondary alexithymia since cognitive therapies are more effective in primary alexithymia than dynamic ones [43]. However, secondary alexithymia responds to a wide range of treatments. Second, due to the small sample size (particularly among different types of FEDs in BPD + FEDs group) and participant selection from a narrow age-range and specific geographical region, the study results should be generalized to other societies with caution. Third, the adults may report different levels of alexithymia in response to certain life events based on the individual difference model. Thus, future studies should focus on some methodological constraints, including sole reliance on self-report measures of alexithymia (due to memory bias and demand characteristics), lack of longitudinal and experimental studies, lack of ethnic differences, limited age range, and negative emotions exclusively.

\section{Conclusions}

In conclusion, this study showed that BPD + FEDs patients found it difficult to identify and describe emotions. However, there was no significant difference in the level of alexithymia among different types of FEDs. The results also suggested that other specified feeding and eating disorders may be the most prevalent FEDs in patients with BPD. Besides, anxiety and depression could mediate the relationship between alexithymia and eating-disordered behaviors in patients with BPD. Accordingly, the FEDs treatment protocols among patients with BPD need to concentrate on emotions and emotion regulation to assess alexithymia, anxiety, and depression conveniently, and determine whether alexithymia precedes FEDs. Nonetheless, further longitudinal and experimental studies with a larger variety of measures to explore psychopathology could contribute to clarifying all these questions and ascertaining primary and secondary alexithymia in FEDs.

\section{Abbreviations}

ANOVA: Analysis of variance; BAI: Beck Anxiety Inventory; BDI-II: Beck Depression Inventory-II; BPD: Borderline personality disorder; BPI: Borderline Personality Inventory; EAT-26: 26-item Eating Attitudes Test; FEDs: Feeding and eating disorders; GHQ-28: 28-item General Health Questionnaire; SCID-5RV: Structured Clinical Interviews for DSM-5: Research Version; SCID-5PD: Structured Clinical Interview for DSM-5 Personality Disorders; TAS-20: 20item Toronto Alexithymia Scale

\section{Acknowledgments}

The author would like to thank the patient, who contributed in conducting the present study.

\section{Author's contributions}

The author read and approved the final manuscript.

\section{Funding}

No funding was received.

\section{Availability of data and materials}

The datasets generated and analyzed during the current study are not publicly available because no consent was obtained from the participants. However, the data are available by the corresponding author on a reasonable request.

\section{Ethics approval and consent to participate}

The study was approved by the ethics committee of the Medical Faculty of the Zahedan University of Medical Sciences (IR.ZAUMS.REC.1398.212). Also, all procedures were according to the latest version of the Declaration of Helsinki. Prior to participation, written informed consent was obtained from all participants and their parents/legal guardians after a comprehensive explanation of the study procedures.

\section{Consent for publication}

Not applicable.

\section{Competing interests}

The author declares that he has no competing interests.

Received: 26 March 2020 Accepted: 17 July 2020

Published online: 17 August 2020

\section{References}

1. Leichsenring F, Leibing E, Kruse J, New AS, Leweke F. Borderline personality disorder. Lancet. 2011;377(9759):74-84. https://doi.org/10.1016/S01406736(10)61422-5.

2. Zanarini MC, Reichman CA, Frankenburg FR, Reich DB, Fitzmaurice G. The course of eating disorders in patients with borderline personality disorder: a 10-year follow-up study. Int J Eat Disord. 2010;43(3):226-32. https://doi.org/ 10.1002/eat.20689.

3. Rosenvinge $\mathrm{JH}$, Martinussen M, Østensen E. The comorbidity of eating disorders and personality disorders: a metaanalytic review of studies published between 1983 and 1998. Eat Weight Disord. 2000;5(2):52-61. https://doi.org/10.1007/bf03327480.

4. Martinussen $M$, Friborg $O$, Schmierer $P$, Kaiser $S$, Øvergård KT, Neunhoeffer $\mathrm{AL}$, et al. The comorbidity of personality disorders in eating disorders: a meta-analysis. Eat Weight Disord. 2017;22(2):201-9. https://doi.org/10.1007/ s40519-016-0345-x.

5. Sansone RA, Sansone LA. Personality pathology and its influence on eating disorders. Innov Clin Neurosci. 2011;8(3):14.

6. Dolan-Sewell, Regina T., Robert F. Krueger, and M. Tracie Shea. Cooccurrence with syndrome disorders. In W. J. Livesley (Ed.), Handbook of personality disorders: Theory, research, and treatment. New York: Guilford Press; 2001.

7. Sloan E, Hall K, Moulding R, Bryce S, Mildred H, Staiger PK. Emotion regulation as a transdiagnostic treatment construct across anxiety, depression, substance, eating and borderline personality disorders: a 
systematic review. Clin Psychol Rev. 2017;57:141-63. https://doi.org/10.1016/ j.cpr.2017.09.002.

8. Berenbaum H. Childhood abuse, alexithymia and personality disorder. J Psychosom Res. 1996;41(6):585-95. https://doi.org/10.1016/s00223999(96)00225-5.

9. Cochrane $C E$, Brewerton TD, Wilson DB, Hodges EL. Alexithymia in the eating disorders. Int J Eat Disord. 1993;14(2):219-22. https://doi.org/10.1002/ 1098-108x(199309)14:2<219::aid-eat2260140212>3.0.co;2-g.

10. Zlotnick C, Mattia Jl, Zimmerman M. The relationship between posttraumatic stress disorder, childhood trauma and alexithymia in an outpatient sample. J Trauma Stress. 2001;14(1):177-88.

11. Wolff S, Stiglmayr C, Bretz HJ, Lammers CH, Auckenthaler A. Emotion identification and tension in female patients with borderline personality disorder. Br J Clin Psychol. 2007;46(3):347-60. https://doi.org/10.1348/ $014466507 \times 173736$.

12. Nowakowski ME, McFarlane T, Cassin S. Alexithymia and eating disorders: a critical review of the literature. J Eat Disord. 2013;1(1):21. https://doi.org/10 1186/2050-2974-1-21.

13. Connelly M, Denney DR. Regulation of emotions during experimental stress in alexithymia. J Psychosom Res. 2007;62(6):649-56. https://doi.org/10.1016/j. jpsychores.2006.12.008

14. Zautra A, Smith B, Affleck G, Tennen H. Examinations of chronic pain and affect relationships: applications of a dynamic model of affect. J Consult Clin Psychol. 2001;69(5):786. https://doi.org/10.1037//0022-006x.69.5.786.

15. Pandey R, Mandal MK. Eysenckian personality dimensions and alexithymia: examining the overlap in terms of perceived autonomic arousal. Personal Individ Differ. 1996;20(4):499-504. https://doi.org/10.1016/01918869(95)00214-6.

16. Eizaguirre $A E$, de Cabezón $A O$, de Alda IO, Olariaga LJ, Juaniz M. Alexithymia and its relationships with anxiety and depression in eating disorders. Personal Individ Differ. 2004;36(2):321-31. https://doi.org/10.1016/S01918869(03)00099-0

17. Westwood H, Kerr-Gaffney J, Stahl D, Tchanturia K. Alexithymia in eating disorders: systematic review and meta-analyses of studies using the Toronto alexithymia scale. J Psychosom Res. 2017;99:66-81. https://doi.org/10.1016/j. jpsychores.2017.06.007.

18. Gillboa-Schechtman E, Avnon L, Zubery E, Jeczmien P. Emotional processing in eating disorders: specific impairment or general distress related deficiency? Depress Anxiety. 2006;23(6):331-9. https://doi.org/10.1002/da. 20163.

19. Forbush KT, South SC, Krueger RF, lacono WG, Clark LA, Keel PK, et al. Locating eating pathology within an empirical diagnostic taxonomy: evidence from a community-based sample. J Abnorm Psychol. 2010;119(2): 282. https://doi.org/10.1037/a0019189.

20. Lane DM, Scott D, Hebl M, Guerra R, Osherson D, Zimmer H. An introduction to statistics. Houston: Rice University; 2017.

21. Taber KS. The use of Cronbach's alpha when developing and reporting research instruments in science education. Res Sci Educ. 2018;48(6):1273-96. https://doi.org/10.1007/s11165-016-9602-2

22. Besharat MA. Reliability and factorial validity of a Farsi version of the 20-item Toronto alexithymia scale with a sample of Iranian students. Psychol Rep. 2007;101 (1):209-20. https://doi.org/10.2466/pro.101.1.209-220.

23. Kaviani H, Mousavi AS. Psychometric properties of the Persian version of Beck anxiety inventory (BAl). Tehran Univ Med J TUMS Publ. 2008;66(2):13640 Persian.

24. Ghassemzadeh H, Mojtabai R, Karamghadiri N, Ebrahimkhani N. Psychometric properties of a Persian-language version of the Beck depression inventory-second edition: BDI-II-PERSIAN. Depress Anxiety. 2005; 21(4):185-92. https://doi.org/10.1002/da.20070

25. Ahmadi S, Moloodi R, Zarbaksh MR, Ghaderi A. Psychometric properties of the eating attitude Test-26 for female Iranian students. Eat Weight Disord. 2014;19(2):183-9. https://doi.org/10.1007/s40519-014-0106-7.

26. Mohammadzadeh A. Validation of the borderline personality inventory in Iran. Int J Behavioral Sci. 2011;5(3):269-77. https://doi.org/10.30491/JBS.2011. 67743.

27. First MB, Williams JB, Benjamin LS, Spitzer RL. User's quide for the SCID-5-PD (structured clinical interview for DSM-5 personality disorder). Arlington: American Psychiatric Association; 2015.

28. First MB, Williams JB, Karg RS, Spitzer RL. User's guide for the SCID-5-CV: structured clinical interview for DSM-5 disorders, clinician version. Arlington: American Psychiatric Association; 2016.
29. Taghavi S. Validity and reliability of the general health questionnaire (GHQ-28) in college students of Shiraz University. J Psychol. 2002;5(4):381-98 Persian.

30. Hayes AF. Introduction to mediation, moderation, and conditional process analysis: a regression-based approach. New York: Guilford Press; 2017.

31. Preacher KJ, Hayes AF. SPSS and SAS procedures for estimating indirect effects in simple mediation models. Behav Res Methods Instrum Comput. 2004;36(4):717-31. https://doi.org/10.3758/bf03206553.

32. Pillay AL, Sargent CA. Relationship of age and education with anxiety, depression, and hopelessness in a South African community sample. Percept Mot Skills. 1999;89(3):881-4. https://doi.org/10.2466/pms.1999.89.3.881.

33. Ta VP, Gesselman AN, Perry BL, Fisher HE, Garcia JR. Stress of singlehood: marita status, domain-specific stress, and anxiety in a national US sample. J Soc Clin Psychol. 2017;36(6):461-85. https://doi.org/10.1521/sscp.2017.36.6.461.

34. Faravelli C, Scarpato MA, Castellini G, Sauro CL. Gender differences in depression and anxiety: the role of age. Psychiatry Res. 2013;210(3):1301-3. https://doi.org/10.1016/.jpsychres.2013.09.027.

35. Carvalhais SM, Lima-Costa MF, Peixoto SV, Firmo JO, Castro-Costa E, Uchoa $E$. The influence of socio-economic conditions on the prevalence of depressive symptoms and its covariates in an elderly population with slight income differences: the Bambui health and aging study (BHAS). Int J Soc Psychiatry. 2008;54(5):447-56. https://doi.org/10.1177/0020764008090792.

36. Bussolotti D, Fernández-Aranda F, Solano R, Jiménez-Murcia S, Turón V, Vallejo J. Marital status and eating disorders: an analysis of its relevance. J Psychosom Res. 2002;53(6):1139-45. https://doi.org/10.1016/s0022 3999(02)00336-7.

37. Hay P, Girosi F, Mond J. Prevalence and sociodemographic correlates of DSM-5 eating disorders in the Australian population. J Eat Disord. 2015;3(1): 19. https://doi.org/10.1186/s40337-015-0056-0.

38. Zanarini MC, Frankenburg FR, Dubo ED, Sickel AE, Trikha A, Levin A, et al. Axis I comorbidity of borderline personality disorder. Am J Psychiatry. 1998; 155(12):1733-9. https://doi.org/10.1176/ajp.155.12.1733.

39. Troop NA, Schmidt UH, Treasure JL. Feelings and fantasy in eating disorders: a factor analysis of the Toronto alexithymia scale. Int J Eat Disord. 1995; 18(2):151-7. https://doi.org/10.1002/1098-108x(199509)18:2<151::aideat2260180207>3.0.co;2-e.

40. Berthoz S, Perdereau F, Godart N, Corcos M, Haviland MG. Observer-and self-rated alexithymia in eating disorder patients: levels and correspondence among three measures. J Psychosom Res. 2007;62(3):341-7. https://doi.org/ 10.1016/j.jpsychores.2006.10.008.

41. Schmidt U, Jiwany A, Treasure J. A controlled study of alexithymia in eating disorders. Compr Psychiatry. 1993;34(1):54-8. https://doi.org/10.1016/0010440x(93)90036-4.

42. Picardi A, Fagnani C, Gigantesco A, Toccaceli V, Lega I, Stazi MA. Genetic influences on alexithymia and their relationship with depressive symptoms. J Psychosom Res. 2011;71(4):256-63. https://doi.org/10.1016/j.jpsychores. 2011.02.016

43. Sexton MC, Sunday SR, Hurt S, Halmi KA. The relationship between alexithymia, depression, and axis II psychopathology in eating disorder inpatients. Int J Eat Disord. 1998;23(3):277-8. https://doi.org/10.1002/ (sici) 1098-108x(199804)23:3<277::aid-eat5>3.0.co;2-g.

\section{Publisher's Note}

Springer Nature remains neutral with regard to jurisdictional claims in published maps and institutional affiliations.

Ready to submit your research? Choose BMC and benefit from

- fast, convenient online submission

- thorough peer review by experienced researchers in your field

- rapid publication on acceptance

- support for research data, including large and complex data types

- gold Open Access which fosters wider collaboration and increased citations

- maximum visibility for your research: over $100 \mathrm{M}$ website views per year

At BMC, research is always in progress.

Learn more biomedcentral.com/submission 